\title{
Intravoxel incoherent motion MRI as a biomarker of sorafenib treatment for advanced hepatocellular carcinoma: a pilot study
}

\author{
Natsuhiko Shirota ${ }^{1}$, Kazuhiro Saito $^{1 *}$, Katsutoshi Sugimoto ${ }^{2}$, Kenichi Takara$^{1}$, Fuminori Moriyasu ${ }^{2}$ \\ and Koichi Tokuuye ${ }^{1}$
}

\begin{abstract}
Background: To evaluate the association between the therapeutic outcomes of sorafenib for advanced hepatocellular carcinoma (HCC) and the parameters of intravoxel incoherent motion (IVIM).

Methods: Nine patients were evaluated prospectively. All patients were Child-Pugh score A. The mean dimension of the lesion was $32 \mathrm{~mm}$ (range: 15-74 mm). MR images were obtained using a 1.5-Tesla superconductive MRI system. Diffusion-weighted imaging was performed under breath-holding using b-values of $0,50,100,150,200,400$, and $800 \mathrm{~s} / \mathrm{mm}^{2}$. The following IVIM parameters were calculated: apparent diffusion coefficient, true diffusion coefficient (DC), pseudo-diffusion coefficient, and perfusion fraction. MRI was performed before treatment and at 1,2, and 4 weeks after beginning treatment. Tumor response at 4 weeks was assessed by CT or MRI using modified RECIST. IVIM parameters of the treatment responders and non-responders were compared.

Results: The DC of responders at baseline was significantly higher than that of the non-responders. The sensitivity and specificity, when a DC of $0.8\left(10^{-3} \mathrm{~mm}^{2} / \mathrm{s}\right)$ or higher was considered to be a responder, were $100 \%$ and $67 \%$, respectively. No significant differences were found in the other parameters between the responders and the non-responders. All IVIM parameters of the responders and non-responders did not change significantly after treatment.
\end{abstract}

Conclusion: The DC before treatment may be a useful parameter for predicting the therapeutic outcome of sorafenib for advanced HCC.

Keywords: Sorafenib, Hepatocellular carcinoma, Intravoxel incoherent motion, Biomarker, Diffusion-weighted imaging

\section{Background}

The multikinase inhibitor sorafenib was reported to prolong the median survival and time to progression of patients with advanced hepatocellular carcinoma (HCC) [1]. Sorafenib inhibits tumor-cell proliferation and tumor angiogenesis [2]. This drug prolongs the stable state of HCC by reducing blood flow to the tumor and by increasing tumor-cell apoptosis, rather than by decreasing

\footnotetext{
*Correspondence: saito-k@tokyo-med.ac.jp

'Department of Radiology, Tokyo Medical University, 6-7-1 Nishishinjuku,

Shinjuku-ku, Tokyo 160-0023, Japan

Full list of author information is available at the end of the article
}

tumor size [1]. However, it was reported that the therapeutic effect of sorafenib could not be accurately evaluated using the Response Evaluation Criteria in Solid Tumors (RECIST) [3], which is conventionally used. Therefore, it has been proposed that the modified RECIST, including the effect on blood flow is more useful for the evaluation of the therapeutic outcomes of cancers $[4,5]$.

On the other hand, other studies have concluded that diffusion-weighted imaging (DWI) was useful for the evaluation of the therapeutic outcomes of advanced $\mathrm{HCC}$, and did not require data on arterial blood flow in 
the lesion [6-8]. Furthermore, it has been proposed that DWI is effective for evaluating the therapeutic outcomes of chemotherapy or radiation therapy on other types of tumors [9-11]. DW images are obtained by visualizing the motion of water molecules randomly using MRI. Because DWI is sensitive to changes of intracellular substances and cell membranes, it is often used for therapeutic monitoring [10]. In previous reports, the bleeding or necrosis of tumors increased diffusion values in the responder group [6].

Lewin et al. reported the usefulness of intravoxel incoherent motion (IVIM) for the evaluation of the therapeutic outcome of sorafenib [7]. It is possible to obtain the true diffusion coefficient reflecting cell density and the perfusion fraction reflecting the microcirculation of tumors using IVIM. Therefore, IVIM may reflect tumor necrosis and neovascular inhibition resulting from the therapeutic effect of sorafenib. Furthermore, IVIM may be able to predict therapeutic outcomes before treatment. Because sorafenib treatment frequently causes side effects and is expensive, it is often difficult for patients to maintain medication compliance. Therefore, it would be highly beneficial if therapeutic efficacy could be determined at an early stage.

Here we reported a pilot study on the efficacy of IVIM for the evaluation of the therapeutic effects and early treatment effects of sorafenib for advanced HCC.

\section{Methods}

The study was approved by the ethics committee of our institution, and written informed consent was obtained from all the patients who participated in this study.

\section{Subjects}

Thirty-seven patients with HCC were examined from July 2009 to January 2012. The study inclusion criteria were patients receiving sorafenib therapy, Barcelona Clinic Liver Cancer stage of B or C, and no contraindications to MRI. This prospective study was part of an assessment of the efficacy of radiological analysis to predict therapeutic outcomes and prognostic expectations. Radiological assessment included contrast-enhanced ultrasound and MRI. Some patients refused all 3 MRI examinations or carelessly forgot to undergo an examination, and 10 patients remained in the study. Of the 10 patients who underwent liver MRI, 1 was excluded because of poor image quality due to artifacts. The final study population included 9 patients with HCC. The largest and previously untreated lesion in each patient was analyzed. Contrast-enhanced ultrasound (US) was performed to evaluate the presence of arterial blood flow in the lesion before baseline MRI. A diagnostic US system (SSA-790A, Aplio XG; Toshiba Medical Systems Corporation, Otawara, Japan) with a $3.75-\mathrm{MHz}$ convex transducer was used. A second-generation US contrast agent (Sonazoid; Daiichi-Sankyo, Tokyo, Japan) was injected as a $0.5-\mathrm{mL}$ bolus into an antecubital vein followed by a $10-\mathrm{mL}$ saline flush at $1 \mathrm{~mL} / \mathrm{s}$.

\section{MRI Protocol}

MR imaging was performed with a 1.5-Tesla scanner 32channel coil system (Avanto, Siemens Medical Systems, Erlangen, Germany) with a peak slew rate of $200 \mathrm{~T} / \mathrm{m} / \mathrm{s}$. MRI sequences were subjected to T1-weighted imaging, T2-weighted imaging, and DWI.

T1-weighted images were acquired using the following sequence parameters: gradient echo sequence; repetition time: $125 \mathrm{~ms}$; dual echo time; opposed phase: $2.38 \mathrm{~ms}$; in phase: $4.76 \mathrm{~ms}$; flip angle: $75^{\circ}$; matrix size: $320 \times 126$; field of view: $400 \times 454 \mathrm{~mm}$; slice thickness: $5 \mathrm{~mm}$; receiver bandwidth: $470 \mathrm{~Hz} /$ pixel; acquisition time: $13 \mathrm{~s}$.

T2-weighted images were acquired during breathholding using the following sequence parameters: turbo spin-echo sequence; repetition time: $3,980 \mathrm{~ms}$; echo time: $95 \mathrm{~ms}$; flip angle: $150^{\circ}$; matrix size: $320 \times 135$; field of view: $400 \times 454 \mathrm{~mm}$; slice thickness: $5 \mathrm{~mm}$; receiver bandwidth: $260 \mathrm{~Hz} /$ pixel; acquisition time: $20 \mathrm{~s}$. DW images were acquired using the following sequence parameters: spin echo based echo-planar imaging; repetition time: $1,200 \mathrm{~ms}$; echo time: $63 \mathrm{~ms}$; flip angle: $90^{\circ}$; matrix size: $110 \times 110$; field of view: $400 \times 454 \mathrm{~mm} ; 1$ averaging; slice thickness: $5 \mathrm{~mm}$; receiver bandwidth: $921 \mathrm{~Hz} /$ pixel; fat suppression; spectral pre-saturation with inversion recovery; acquisition time: $20 \mathrm{~s} ; b$-values: $0,50,100,150$, 200, 400, and $800 \mathrm{~s} / \mathrm{mm}^{2}$. DWI was performed during breath-holding.

Tumors were evaluated by MRI at baseline, and at 1 , 2 , and 4 weeks after sorafenib treatment.

\section{Follow-up}

CT or MRI was performed before the start of sorafenib therapy, at 1 month and every 2 months thereafter. Dynamic CT was performed using either a 16-detector row or 64-detector row CT scanner. Iohexol 300 (Omnipaque 300, Daiichi-Sankyo) was injected over $30 \mathrm{~s}$ [12]. The amount of contrast agent used was $600 \mathrm{mgI} / \mathrm{kg}$ [13]. The arterial-dominant phase was obtained using a monitor scan; following this the portal-dominant phase and equilibrium phase were obtained. Dynamic MRI was performed using gadoterate meglumine (Magnescope, Guerbet) or gadolinium-ethoxybenzyl-diethylenetriamine pentaacetic acid (Primovist, Bayer). Magnescope $(0.1 \mathrm{mmol} / \mathrm{kg})$ was injected at $2 \mathrm{~mL} / \mathrm{s}$ and Primovist $(0.025 \mathrm{mmol} / \mathrm{kg})$ was injected at $1 \mathrm{~mL} / \mathrm{s}$. Monitor scan was performed by first obtaining the arterial-dominant phase and then the portaldominant and equilibrium phases. We evaluated the curative effects using dynamic CT or dynamic MRI at baseline, and after 1, 2, and 4 weeks of sorafenib treatment. We 
evaluated the curative effect by modified RECIST [3]. Curative effects were divided into 2 groups, namely, responders (complete response, partial response, and stable disease) and non-responders (progressive disease).

\section{Changes in signal strength}

Changes in signal strength of the lesions on T1weighted and T2-weighted imaging were evaluated by 2 radiologists who had 2 and 24 years of experience, by consensus reading. They compared the signal strengths and homogeneity of the lesions at baseline with those after 1,2, and 4 weeks of sorafenib treatment, and they recorded whether a difference was present or absent.

\section{Calculation of IVIM parameters}

The IVIM model is considered to provide the pure molecular diffusion (D) separately from the blood microcirculation (proportion of blood microcirculation [PF] and pseudo-diffusion coefficient [D*]), when multiple $b$ values are obtained, from low $b$-values $\left(<200 \mathrm{~s} / \mathrm{mm}^{2}\right)$ to high $b$-values $\left(>200 \mathrm{~s} / \mathrm{mm}^{2}\right)$ [14]. IVIM parameters were calculated using the following formula [14]:

$$
\mathrm{S}_{\mathrm{b}} / \mathrm{S}_{0}=\mathrm{f} \times \exp -\{(\mathrm{D} *+\mathrm{D}) \times \mathrm{b}\}+(1-\mathrm{f}) \times\{-\mathrm{D} \times \mathrm{b}\}
$$

D: true diffusion coefficient (DC); D*: pseudo-diffusion coefficient; f: perfusion fraction (PF); $\mathrm{S}_{\mathrm{b}}, \mathrm{S}_{0}$ : signal intensity with and without the application of the diffusion gradient, respectively.

The 2-step fitting procedure was adopted to determine $\mathrm{PF}, \mathrm{D}$, and $\mathrm{D}^{*}$, required because of the high dispersion and limited sampling of DWI signals at low $b$-values $(b<$ $200 \mathrm{~s} / \mathrm{mm}^{2}$ ). Values of D were estimated from signal intensity data at high $b$-values $\left(b>200 \mathrm{~s} / \mathrm{mm}^{2}\right)$. Considering that $\mathrm{D}^{*}$ is significantly greater than $\mathrm{D}$, its effect on signal decay can be neglected for $b$-values greater than $200 \mathrm{~s} /$ $\mathrm{mm}^{2}$. Eq. (1) can be simplified and the D can be obtained using only $b$-values equal to or greater than $200 \mathrm{~s} / \mathrm{mm}^{2}$, with the following simple linear fit equation:

$$
\mathrm{S}_{\mathrm{b}} / \mathrm{S}_{0}=\exp (-\mathrm{bD})
$$

After determination of the D-value using Eq. (2), PF and $\mathrm{D}^{*}$ can be processed using a nonlinear least squares estimate based on Eq. (1). The apparent diffusion coefficient (ADC) was obtained using all $b$-values by simple linear fitting as in Eq. (2). IVIM parameters were calculated using freely available software at the website (http://yamarad.umin.ne.jp/ivim/simplex.html). The IVIM data were constrained as follows: $0<\mathrm{PF}<1,0<\mathrm{D}<\mathrm{D}^{*}<$ $1 \mathrm{~mm}^{2} / \mathrm{s}, 0<$ ADC.

\section{Statistical analysis}

IVIM parameters were expressed as means \pm standard deviations. Changes in the signal strengths of T1weighted and T2-weighted images of the responder group and the non-responder group were statistically analyzed using the chi-squared test. The differences in IVIM parameters between the responder group and the non-responder group were statistically analyzed using the Mann-Whitney $U$ test and Fisher exact test. In addition, differences in IVIM parameters between pretreatment and post-treatment were evaluated using the Friedman test. A $p$-value less than 0.05 was considered to indicate a statistically significant difference between 2 groups. When a significant difference was observed, the cut-off value was determined by receiver operating characteristic analysis, and then sensitivity and specificity were calculated. All statistical analyses were performed using SPSS statistics software (version 22, SPSS) for Microsoft windows.

\section{Results}

Six patients (67 \%) had at least 1 non-permissible value of PF or $\mathrm{D}^{*}$ within the 4 consecutive examinations. Six patients were classified as responders (Complete Response: 1; Stable Disease: 5), and 3 patients were classified as non-responders. Detailed information of the patients is described in Table 1. The sizes of the lesions did not significantly change in both the responder and non-responder group, although the lesions in the nonresponder group tended to increase in size. There were no remarkable signal changes between before and after treatment on T1-weighted and T2-weighted imaging in both the responders and the non-responders.

The IVIM parameters of ADC, D, D*, and PF in the responders and non-responders at baseline, and after 1 , 2 , and 4 weeks of treatment are shown in Table 2 and Figs. 1, 2, 3 and 4.

The ADCs of the responders at baseline, and after 1,2, and 4 weeks of treatment were $1.06 \pm 0.21,0.98 \pm 0.25$, $0.93 \pm 0.16$, and $0.84 \pm 0.11\left(10^{-3} \mathrm{~mm}^{2} / \mathrm{s}\right)$, respectively, and of the non-responders were $0.85 \pm 0.04,1.00 \pm 0.05$, $0.88 \pm 0.33$, and $0.92 \pm 0.33\left(10^{-3} \mathrm{~mm}^{2} / \mathrm{s}\right)$, respectively. A statistically significant difference was not detected between the responders and the non-responders. $(p=$ $0.095,0.548,1.00$, and 0.905 at baseline, and after 1, 2, and 4 weeks of treatment, respectively). The DC of the responders at baseline, and after 1,2 , and 4 weeks of treatment were $1.04 \pm 0.23,0.99 \pm 0.38,0.90 \pm 0.25$, and $0.80 \pm 0.08\left(10^{-3} \mathrm{~mm}^{2} / \mathrm{s}\right)$, respectively, and of the nonresponders were $0.78 \pm 0.06,0.94 \pm 0.20,0.84 \pm 0.32$, and $0.83 \pm 0.23\left(10^{-3} \mathrm{~mm}^{2} / \mathrm{s}\right)$, respectively. The DC of responders at baseline was significantly higher than that of the non-responders $(p=0.048,1.00,0.905$, and 0.714 at baseline, and after 1,2 , and 4 weeks of treatment, 
Table 1 Patient characteristics

\begin{tabular}{|c|c|c|}
\hline & Responder & Non-responder \\
\hline Age (yrs; mean \pm SD) & $72.2 \pm 2.7$ & $61.3 \pm 13.1$ \\
\hline \multicolumn{3}{|l|}{ Sex } \\
\hline Men & 5 & 2 \\
\hline Women & 1 & 1 \\
\hline \multicolumn{3}{|l|}{ Cause of disease } \\
\hline Hepatitis B & 0 & 1 \\
\hline Hepatitis C & 6 & 2 \\
\hline \multicolumn{3}{|l|}{$\mathrm{BCLC}$ stage } \\
\hline B & 4 & 1 \\
\hline C & 2 & 2 \\
\hline \multicolumn{3}{|l|}{ Previous therapy } \\
\hline Surgery & 1 & 0 \\
\hline TAE/TACE/RFA & 4 & 2 \\
\hline None & 1 & 1 \\
\hline \multicolumn{3}{|c|}{ Tumor size (mm; mean $\pm \mathrm{SD})$} \\
\hline Baseline & $30.7 \pm 22.1$ & $33.5 \pm 14.1$ \\
\hline 1 week & $30.3 \pm 21.8$ & $35.0 \pm 14.6$ \\
\hline 2 weeks & $31.0 \pm 20.7$ & $37.3 \pm 16.7$ \\
\hline 4 weeks & $31.5 \pm 19.4$ & $38.5 \pm 15.9$ \\
\hline \multicolumn{3}{|l|}{ Treatment } \\
\hline Sorafenib (initial dose) & 200 mg twice daily & 200 mg twice daily \\
\hline
\end{tabular}

respectively). The sensitivity and specificity, when a DC of $0.8\left(10^{-3} \mathrm{~mm}^{2} / \mathrm{s}\right)$ or higher was considered to be a responder, were $100 \%$ (95 \% CI: 83, 100) and $67 \%$ (95\% CI: 27.2, 66.7), respectively. The D*s of responders at baseline, and after 1,2 , and 4 weeks of treatment were $228 \pm 392, \quad 452 \pm 376, \quad 470 \pm 441$, and $321 \pm 389\left(10^{-3}\right.$ $\mathrm{mm}^{2} / \mathrm{s}$ ), respectively, and of the non-responders were $317 \pm 274, \quad 657 \pm 555, \quad 676 \pm 272$, and $323 \pm 276\left(10^{-3}\right.$ $\left.\mathrm{mm}^{2} / \mathrm{s}\right)$, respectively. There were no significant differences between the 2 groups $(p=0.381,0.714,0.548$, and 1.00 at baseline, and after 1,2 , and 4 weeks of treatment, respectively). The PFs of the responders at baseline, and after 1,2 , and 4 weeks of treatment were $0.20 \pm 0.28$, $0.06 \pm 0.04,0.12 \pm 0.11$, and $0.08 \pm 0.06\left(10^{-3} \mathrm{~mm}^{2} / \mathrm{s}\right)$, respectively, and of the non-responders were $0.10 \pm 0.08$, $0.06 \pm 0.04,0.07 \pm 0.08$, and $0.10 \pm 0.12\left(10^{-3} \mathrm{~mm}^{2} / \mathrm{s}\right)$, respectively. There were no significant differences between the 2 groups ( $p=0.905,1.00,0.905$, and 1.00 at baseline, and after 1, 2, and 4 weeks of treatment, respectively).

Regarding changes in each parameter with treatment, the responders showed a decrease in the ADC and DC, whereas the non-responders did not. However, these changes were not statistically significant $(p=0.102$ and 0.719 for $\mathrm{ADC}$, and $p=0.100$ and 0.334 for $\mathrm{DC}$ in the responder and non-responder group, respectively). The responders showed a decrease in PF at 1 week after treatment compared with the baseline, but this was not statistically significant $(p=0.978$ and 0.801 in the responder and non-responder group, respectively). Furthermore, we did not observe a statistically significant difference in $\mathrm{D}^{*}(p=0.261$ and 0.801 in the responder and non-responder group, respectively).

\section{Discussion}

In our study, DC values in the responder group were significantly higher than those in the non-responder group at baseline, suggesting that it is possible to predict therapeutic outcome before the initiation of treatment. In addition, at baseline, ADC values of the responder group were higher than those of the non-responder group, although this difference was not significant. Woo et al. reported that the histological grade of HCC correlated more strongly with the DC than the ADC [15]. Because the ADC includes not only pure diffusion, but also perfusion as compared with $\mathrm{DC}$, its interpretation may be complicated. They reported that the DC of patients with high-grade $\mathrm{HCC}$ was significantly lower than that of patients with low-grade HCC. It was also reported that favorable treatment results were obtained with sorafenib in patients with histologically well-differentiated tumors. Furthermore, the degrees of differentiation of tumors were shown to correlate with their expression levels of vascular endothelial growth factor (VEGF), i.e., high expression levels of VEGF indicated welldifferentiated HCC [16]. We believe that the results of our present study reflect the results of this previous study [17].

Table 2 IVIM parameters of responders and non-responders

\begin{tabular}{|c|c|c|c|c|c|c|c|c|}
\hline & \multicolumn{2}{|c|}{$\mathrm{ADC}\left(10^{-3} \mathrm{~mm}^{2} / \mathrm{s}\right)$} & \multicolumn{2}{|c|}{$\mathrm{DC}\left(10^{-3} \mathrm{~mm}^{2} / \mathrm{s}\right)$} & \multicolumn{2}{|c|}{$\mathrm{D}^{*}\left(10^{-3} \mathrm{~mm}^{2} / \mathrm{s}\right)$} & \multicolumn{2}{|l|}{ PF } \\
\hline & Responder & Non-responder & Responder & Non-responder & Responder & Non-responder & Responder & Non-responder \\
\hline Baseline & $1.06 \pm 0.21$ & $0.85 \pm 0.04$ & $1.04 \pm 0.23$ & $0.78 \pm 0.06$ & $228 \pm 392$ & $317 \pm 274$ & $0.20 \pm 0.28$ & $0.10 \pm 0.08$ \\
\hline 1 week & $0.98 \pm 0.25$ & $1.00 \pm 0.05$ & $0.99 \pm 0.38$ & $0.94 \pm 0.20$ & $452 \pm 376$ & $657 \pm 555$ & $0.06 \pm 0.04$ & $0.06 \pm 0.04$ \\
\hline 2 weeks & $0.93 \pm 0.16$ & $0.88 \pm 0.33$ & $0.90 \pm 0.25$ & $0.84 \pm 0.32$ & $470 \pm 441$ & $676 \pm 272$ & $0.12 \pm 0.11$ & $0.07 \pm 0.08$ \\
\hline 4 weeks & $0.84 \pm 0.11$ & $0.92 \pm 0.33$ & $0.80 \pm 0.08$ & $0.83 \pm 0.23$ & $321 \pm 389$ & $323 \pm 276$ & $0.08 \pm 0.06$ & $0.10 \pm 0.12$ \\
\hline
\end{tabular}

$A D C$ apparent diffusion coefficient, $D C$ true diffusion coefficient, $D^{*}$ pseudo-diffusion coefficient, $P F$ perfusion fraction 


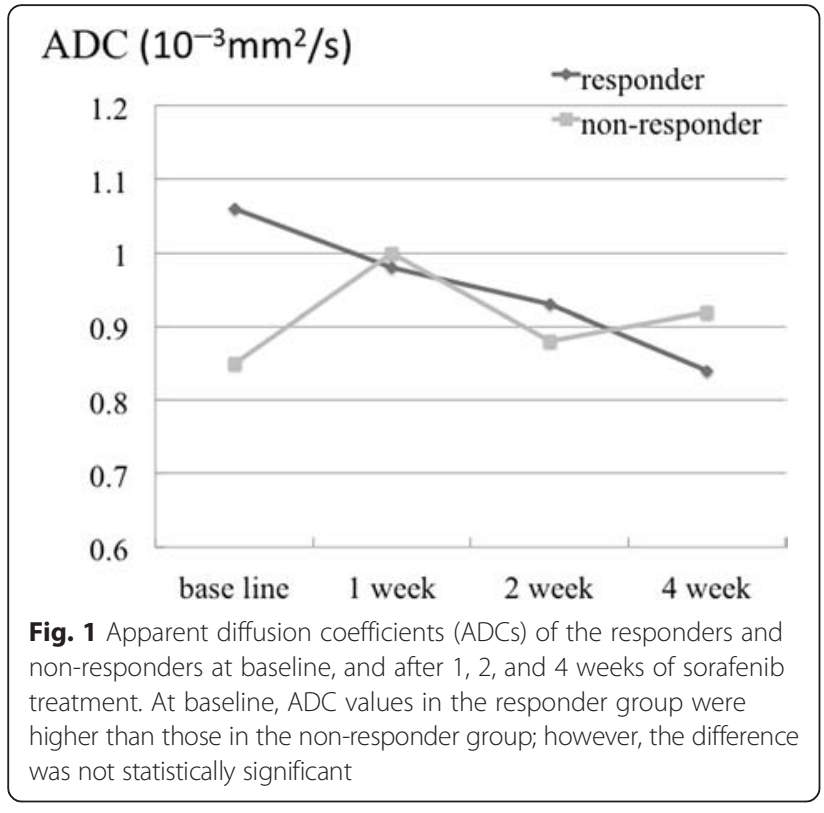

The DC and ADC values showed a decrease in the responders with treatment, but this was not significant, and is consistent with previous reports [6, 7]. Schraml et al. reported that these changes are caused by bleeding [5]; however, we did not detect any bleeding, consistent with the report by Lewin et al. [7]. They reported that the ADC was increased at 2-3 months after treatment because of necrotic changes. We did not find any obvious changes on the images, such as those reflecting necrosis, because we only evaluated the patients up to 4 weeks of treatment.

We observed a decrease in the PF after 1 week of treatment in the responders. On the other hand, Lewin

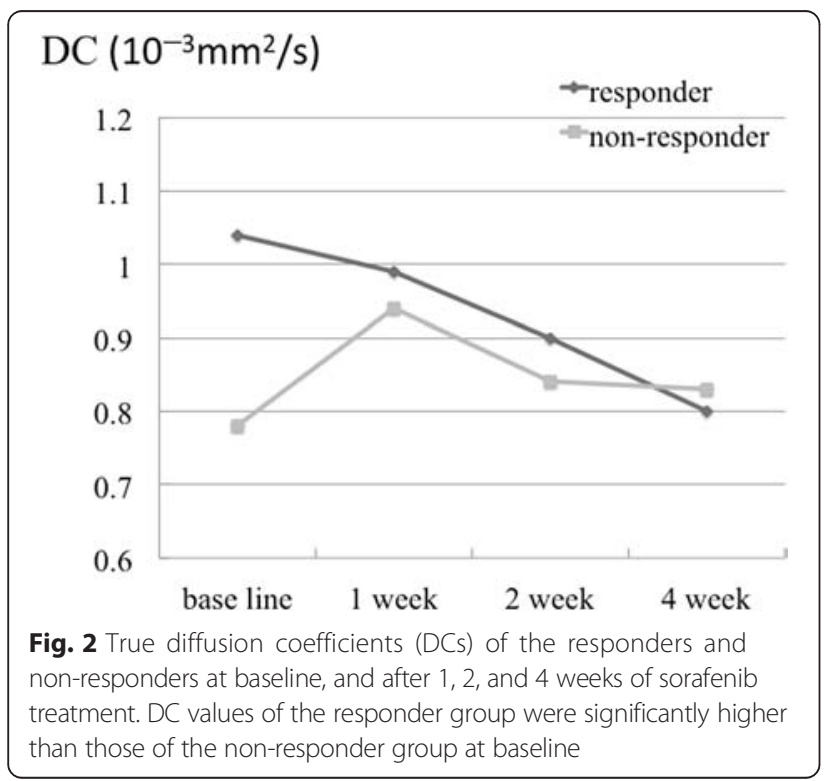

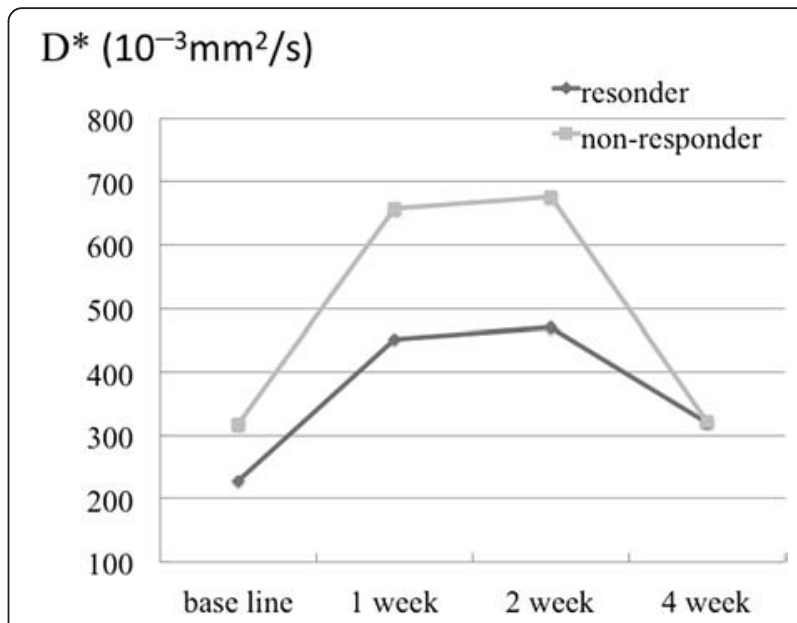

Fig. 3 Pseudo-diffusion coefficients of the responders and nonresponders at baseline, and after 1, 2, and 4 weeks of treatment. No significant changes or differences were observed between the 2 groups

et al. reported an increase in the PF after 2 weeks of treatment [7]. Because sorafenib inhibits tumor angiogenesis, it causes the disruption and normalization of tumor vessels [18]. This normalization of tumor blood vessels suppresses permeability, resulting in a decrease in the pressure of the tumor tissue. Lewin et al. described the cause of the increased PF as an increase in the perfusion rate by normalization of tumor blood vessels. In our present study, the factor that differed from the study of Lewin et al. was the scanning periods. In addition, the method of calculation of the PFs was also different. It has been reported that PFs and D*s have poor reproducibility [19]. Therefore, it may be useful to scan many low $b$-values to obtain a stable PF value. We

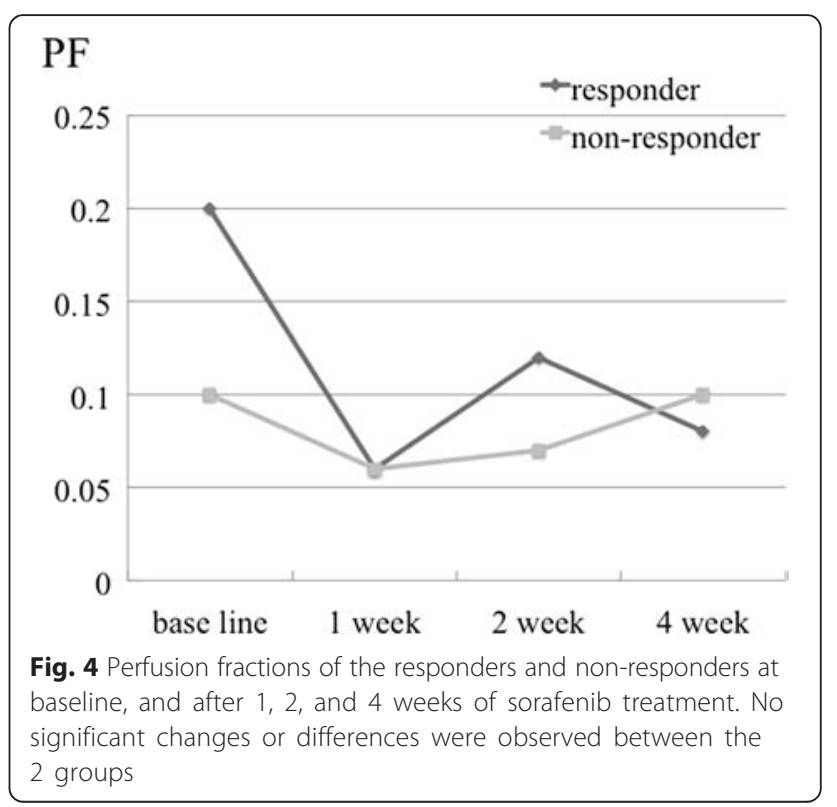


measured a total of $7 b$-values $(0,50,100,150,200,400$, and $800 \mathrm{~s} / \mathrm{mm}^{2}$ ), whereas Lewin et al. measured a total of $4\left(0,200,400\right.$, and $\left.800 \mathrm{~s} / \mathrm{mm}^{2}\right)$. In this regard, our results may be more reliable.

Our study has several limitations. The first limitation is the small number of patients studied. High sensitivity and specificity of differentiation between responders and non-responders was found in our study. However, these results might be an overestimation because of the small number of subjects. The study should be repeated with a larger number of patients in the future. The second limitation is that some cases showed poor fitting of IVIM. When such cases occurred, outliers were removed from the measurements. In other studies, scanning by the appropriate $b$-values and other techniques, such as Bayesian fitting, were used to obtain better fitting $[20,21]$. In the future, other methods to improve the fitting should be tested. The third limitation is the lack of evaluation of reproducibility. A previous report stated that $\mathrm{D}^{*}$ and $\mathrm{PF}$ showed poor reproducibility, whereas DC showed relatively high reproducibility [19]. Therefore, we believe that the conclusion of our study is reliable. The fourth limitation is that we used breath-holding DWI. This technique is faster than respiratory-triggered DWI but has the problem of a lower signal-to-noise ratio. However, Kim et al. reported that ADCs calculated from breath-holding DWI were more reproducible than those from respiratorytriggered DWI [22]. Furthermore, respiratory-triggered DWI requires longer acquisition times, and is prone to misregistration, potentially leading to an inaccurate ADC map [23]. Therefore, we believe that the breathholding technique was adequate for performing routine examinations.

\section{Conclusions}

In conclusion, our results suggest that the DC obtained by IVIM MRI may be useful as a biomarker for predicting the therapeutic effects of sorafenib for HCC.

\section{Competing interests}

The authors declare that they have no financial disclosures and competing interests.

\begin{abstract}
Authors' contributions
K. Saito, K. Sugimoto, F. Moriyasu, and K. Tokuuye conceived of the study, and participated in its design and coordination. N. Shirota and K. Saito performed the image analyses, and K. Saito and K. Takara performed the statistical analyses. All authors read and approved the final manuscript.
\end{abstract}

\section{Author details}

'Department of Radiology, Tokyo Medical University, 6-7-1 Nishishinjuku, Shinjuku-ku, Tokyo 160-0023, Japan. ${ }^{2}$ Department of Gastroenterology and Hepatology, Tokyo Medical University, Shinjuku-ku, Tokyo, Japan.

Received: 25 September 2015 Accepted: 24 January 2016

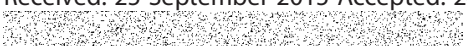

\section{References}

1. Llovet JM, Ricci S, Mazzaferro V, Hilgard P, Gane E, Blanc JF, et al. Sorafenib in advanced hepatocellular carcinoma. N Engl J Med. 2008;359:378-90.

2. Wilhelm S, Carter C, Lynch M, Lowinger T, Dumas J, Smith RA, et al. Discovery and development of sorafenib: a multikinase inhibitor for treating cancer. Nat Rev Drug Discov. 2006;5:835-44.

3. Lencioni R, Llovet JM. Modified RECIST (mRECIST) assessment for hepatocellular carcinoma. Semin Liver Dis. 2010;30:52-60.

4. Spira D, Fenchel M, Lauer UM, Claussen CD, Gregor M, Bitzer M, et al. Comparison of different tumor response criteria in patients with hepatocellular carcinoma after systemic therapy with the multikinase inhibitor sorafenib. Acad Radiol. 2011;18:89-96.

5. Goh V, Sarker D, Osmany S, Cook GJ. Functional imaging techniques in hepatocellular carcinoma. Eur J Nucl Med Mol Imaging. 2012;39:1070-9.

6. Schraml C, Schwenzer NF, Martirosian P, Bitzer M, Lauer U, Claussen CD, et al. Diffusion-weighted MRI of advanced hepatocellular carcinoma during sorafenib treatment: initial results. Am J Roentgenol. 2009;193:W301-307.

7. Lewin M, Fartoux L, Vignaud A, Arrive L, Menu Y, Rosmorduc O. The diffusion-weighted imaging perfusion fraction $\mathrm{f}$ is a potential marker of sorafenib treatment in advanced hepatocellular carcinoma: a pilot study. Eur Radiol. 2011;21:281-90.

8. Kamel IR, Bluemke DA, Eng J, Liapi E, Messersmith W, Reyes DK, et al. The role of functional MR imaging in the assessment of tumor response after chemoembolization in patients with hepatocellular carcinoma. J Vasc Interv Radiol. 2006;17:505-12.

9. Dzik-Jurasz A, Domenig C, George M, Wolber J, Padhani A, Brown G, et al. Diffusion MRI for prediction of response of rectal cancer to chemoradiation. Lancet. 2002;360:307-8.

10. Koh DM, Collins DJ. Diffusion-weighted MRI in the body: applications and challenges in oncology. Am J Roentgenol. 2007;188:1622-35.

11. Mardor Y, Roth Y, Ochershvilli A, Spiegelmann R, Tichler T, Daniels D, et al. Pretreatment prediction of brain tumors' response to radiation therapy using high b-value diffusion-weighted MRI. Neoplasia. 2004;6:136-42.

12. Yanaga Y, Awai K, Nakaura T, Namimoto T, Oda S, Funama Y, et al. Optimal contrast dose for depiction of hypervascular hepatocellular carcinoma at dynamic CT using 64-MDCT. Am J Roentgenol. 2008;190:1003-9.

13. Yamashita Y, Komohara Y, Takahashi M, Uchida M, Hayabuchi N, Shimizu T, et al. Abdominal helical CT: evaluation of optimal doses of intravenous contrast material-a prospective randomized study. Radiology. 2000;216:718-23.

14. Le Bihan D, Breton E, Lallemand D, Aubin ML, Vignaud J, Laval-Jeantet M. Separation of diffusion and perfusion in intravoxel incoherent motion MR imaging. Radiology. 1988;168:497-505.

15. Woo S, Lee JM, Yoon JH, Joo I, Han JK, Choi Bl. Intravoxel incoherent motion diffusion-weighted MR imaging of hepatocellular carcinoma: correlation with enhancement degree and histologic grade. Radiology. 2014;270:758-67.

16. Yamaguchi R, Yano H, lemura A, Ogasawara S, Haramaki M, Kojiro M. Expression of vascular endothelial growth factor in human hepatocellular carcinoma. Hepatology. 1998;28:68-77.

17. Takeda H, Nishikawa H, Osaki Y, Tsuchiya K, Joko K, Ogawa C, et al. Clinical features associated with radiological response to sorafenib in unresectable hepatocellular carcinoma: a large multicenter study in Japan. Liver Int. 2015; 35:1581-9.

18. Jain RK. Normalization of tumor vasculature: an emerging concept in antiangiogenic therapy. Science. 2005;307:58-62.

19. Andreou A, Koh DM, Collins DJ, Blackledge M, Wallace T, Leach MO, et al. Measurement reproducibility of perfusion fraction and pseudodiffusion coefficient derived by intravoxel incoherent motion diffusion-weighted MR imaging in normal liver and metastases. Eur Radiol. 2013;23:428-34.

20. Neil JJ, Bretthorst GL. On the use of Bayesian probability theory for analysis of exponential decay data: an example taken from intravoxel incoherent motion experiments. Magn Reson Med. 1993:29:642-7.

21. Luciani A, Vignaud A, Cavet M, Nhieu JT, Mallat A, Ruel L, et al. Liver cirrhosis: intravoxel incoherent motion MR imaging-pilot study. Radiology. 2008;249:891-9.

22. Kim SY, Lee SS, Park B, Kim N, Kim JK, Park SH, et al. Reproducibility of measurement of apparent diffusion coefficients of malignant hepatic tumors: effect of DWI techniques and calculation methods. J Magn Reson Imaging. 2012;36:1131-8.

23. Ichikawa T, Arbab AS, Araki T, Touyama K, Haradome H, Hachiya J, et al. Perfusion MR imaging with a superparamagnetic iron oxide using T2-weighted and susceptibility-sensitive echoplanar sequences: evaluation of tumor vascularity in hepatocellular carcinoma. Am J Roentgenol. 1999;173:207-13. 